\section{Diakonie und 500 Jahre Reformation in Zürich}

«Ein Christ syn ist nit schwätzen von Christo, sunder wandlen, wie er gewandelt hat. Ein Christ syn ist der schönst und zierlichest Adel, der in dem Himmel und uff erden sin mag.»

(Ulrich Zwingli) $^{\gamma}$

Christoph Sigrist

\section{Einleitung: Schattenwurf Zwingli}

«Bildung und Armut: Beruf erlernen und Armut bekämpfen. Wer einen Beruf erlernt, beruft sich darauf, eigenverantwortlich das Heft in die Hand zu nehmen und solidarisch für andere Verantwortung zu übernehmen. Die Hilfe zur Selbsthilfe verhilft, selber zu denken und eigene Schritte zu wirtschaftlicher Unabhängigkeit zu wagen. Was die Reformatoren mit ihren Bildungsprogrammen zur Armutsbekämpfung vorgelegt haben, ermutigt, in Zukunft noch mehr in Gott's Names zu investieren in Bildung von Sprache und Beruf gegen Armut, Abhängigkeit und Ausgrenzung. In Gottes Namen? Ja, denn Gott ist - so die Überzeugung

1 Huldreich Zwingli, Sämtliche Werke III, hg. von Emil Egli et al., Corpus Reformatorum 88-108, Leipzig/Zürich 1905-2013, 407. Diese Ende 1524 erschienene Schrift nimmt die sozialen Umwälzungen und Unsicherheiten mitten im Transformationsprozess in Zürich auf (Räumung der Kirchen, Aufruhr auf dem Land mit Aufbrechen der Täufer-Verfolgung und der Zins-Fragen). Der zitierte Satz steht im Zusammenhang mit 1. Kor 14,26 und betont die Wirkung des Gottesdienstes zur Erbauung von Gemeinde und damit auch zur christlicher Gemeinschaft.
Zwinglis -, nicht neutral, sondern parteiisch für die Elenden, Witwen und Waisen.»

Mit diesen Worten versuchte ich im Reformationsjahr am 1. Februar 2017 die Armutsdebatte von damals mit der Frage nach der Bildung von heute zu verbinden. Zusammen mit Pfrn. Catherine Mc Millan erfülle ich seit November 2015 das Mandat der Evang.-ref. Kirche des Kantons Zürich, als Botschafter die Erinnerung an die reformatorischen Aufbrüche mit dem gesellschaftlichen und kirchlichen Leben von heute in einen schöpferischen und prospektiven Zusammenhang zu bringen. Der «Schattenwurf Zwingli» war ein Versuch, während eines Jahres an jedem ersten Tag im Monat den Dreiklang «Botschaft - Kunstinstallation Debatte» in der Stadt Zürich hörbar werden zu lassen. Zur inhaltlichen Botschaft, jeweils in der Neuen Zürcher Zeitung (NZZ) veröffentlicht, projizierte Gerry Hofstetter als Künstler seine Lichtbilder mit dem Schatten Zwinglis an Kirchen, Ratshäuser und Museen. Der Schatten Zwinglis ist aus dem Lichtspiel geformt, das abends von der Statue von Ulrich Zwingli (1885 zum Anlass des 400. Geburtstags eingeweiht) vor der Wasserkirche durch die Strassenlampen an die Kirchenmauer aufscheint. Die Disputationen über das Thema mit Gästen aus unterschiedlichen Lebenswelten vertiefte die Botschaft mit aktuellem Bezug. Den Dreiklang trugen wir im Verlauf des Jahres wie eine reformatorische Fackel entlang der biografischen Achse Ulrich Zwinglis durch Länder und zu den Leuten. Mit diesem Konzept wurden drei grundlegende Impulse der Zürcherischen Reformation aufgenommen: Am 1. Januar 1519 begann Zwingli erstens mit seiner Predigttätigkeit im Grossmünster als neu gewählter Leutpriester, indem er die agendarische Struktur der Homilie in der Messe mit dem bis heute als reformiertes Prinzip im Grossmünster hochgehaltenen Konzept der lectio continua, der fortlau-

2 Christoph Sigrist / Gerry Hofstetter / Alexandra Steinegger (Hg.), Schattenwurf Zwingli. 500 Jahre Reformation in der Schweiz, Zürich 2018, 32.
Jahrbuch Diakonie Schweiz 2 (2018) - ISSN 2504-3994

Dieser Text ist lizenziert unter einer Creative Commons Namensnennung 4.0 International Lizenz (CC BY 4.0): (https://creativecommons.org/licenses/by/4.0/).
Jahrbuch Diakonie Schweiz 2 (2018) http://dx.doi.org/10.22018/JDS.2018.9 
fenden Auslegung des Matthäusevangeliums, durchbrach. Zum Zweiten erwiesen sich die öffentlichen Disputationen im Ratshaus oder Grossmünster als wichtigstes kommunikatives Instrument in der Umsetzung der Reformation. Drittens zeigt die kontrovers diskutierte übergrosse Statue des Reformators mit Bibel und Schwert in der Hand die Zwiespältigkeit kontextueller Interpretation des reformatorischen Erbes mit dem jeweiligen Zeitgeist. Der Schatten des Kulturkampfes in der neu gegründeten Schweiz des 19. Jahrhunderts bricht sich an den Herausforderungen einer plural gewordenen Gesellschaft - d.h. einer Gesellschaft, in der nicht mehr die konfessionellen Grabenkämpfe das kulturelle und politische Leben prägen, sondern die Frage, wie das Christsein selber angesichts der multikulturellen und interreligiösen Zusammensetzung von Menschen, Gütern und sozialen und gesellschaftspolitischen Systemen zu gestalten ist.

Der vorliegende Beitrag nimmt diese aktuelle Herausforderung auf, indem er den Fokus auf die Frage legt, wie denn heute das reformatorische Erbe mit Blick auf den diakonischen Auftrag von Kirchen und Werken fruchtbar und zukunftsgerichtet, zielführend und nachhaltig zu verstehen ist. Das Bedürfnis, Diakonie im Lichte der Reformation heute $\mathrm{zu}$ interpretieren, ist angesichts der Feierlichkeiten in Deutschland zum Luther-Jahr 2017 von Johannes Eurich breit entfaltet worden. ${ }^{3}$ Aus Schweizerischer Perspektive soll ausgehend vom diakonischen Konzept, wie Heinz Rüegger und ich es mit grosser, kontrovers diskutierter Wirkung im deutschsprachigen Raum entworfen haben, ${ }^{4}$ das reformatorische Wirken vor allem von Ulrich Zwingli mit Blick auf den diakoni-

3 Sozialwissenschaftliches Institut der EKD (Hg.), Diakonie. Reformation heute, Hannover 2016.

4 Heinz Rüegger / Christoph Sigrist, Diakonie - eine Einführung, Zürich 2011; Christoph Sigrist / Heinz Rüegger (Hg.), Helfendes Handeln im Spannungsfeld theologischer Begründungsansätze, Zürich 2014. schen Auftrag reflektiert und auf die Wirkungen aktueller Herausforderungen hin befragt werden.

Dass eine Ausweitung der diakonischen Reflexionen auf den Schweizerischen Kontext notwendig ist, macht der Beitrag von Ute Gaus im soeben erschienenen Diakonie-Lexikon, dem grundlegenden Werk für diakonisches Handeln im deutschsprachigen Raum, deutlich. Sie blendet bei der Reflexion von Diakonie und Reformation (mit Ausnahme des Hinweises auf das reformierte Diakonenamt Johannes Calvins) den Schweizerischen Kontext schlicht aus. ${ }^{5}$ Andere Beiträge gehen erfreulicherweise spezifisch auf die Schweizerische Situation ein: Michael Klein macht mit Blick auf den Beitrag der protestantischen Theologie die soziale und politische Situation in Zürich öffentlich; ${ }^{6}$ Thomas Hörnig integriert im eben erschienenen Grundlagenbuch Diakonik die Schweizerische perspektive; ${ }^{7}$ und im Beiträg von Thomas Kuhn wird m.W. zum ersten Mal in der Darstellung der Anfänge der Diakonie in der Neuzeit der Fokus auf die Situation Zürich mit seiner Diakonie gelegt. ${ }^{8}$

5 Ute Gaus, Art. Reformation, in: Norbert Friedrich u.a. (Hg.), Diakonie-Lexikon, Neukirchen 2016, $361 \mathrm{f}$.

6 Michael Klein, Der Beitrag der protestantischen Theologie zur Wohlfahrtstätigkeit im 16. Jahrhundert, in: Theodor Strohm / ders. (Hg.), Die Entstehung einer sozialen Ordnung Europas, Bd. 1. Historische Studien und exemplarische Beiträge zur Sozialreform im 16. Jahrhundert, Heidelberg 2004, 146-179 (160-166); zur Quelle: Michae Klein, Almosenordnung der Stadt Zürich 1525, in: Theodor Strohm / ders. (Hg.), Die Entstehung einer sozialen Ordnung Europas, Bd.2 Europäische Ordnungen zur Reform der Armenpflege im 16. Jahrhundert, Heidelberg 2004, 100-107.

7 Thomas Hörnig, 2. Geschichte der Diakonie - ein kritischer Zugang aus der Armutsperspektive, in: Johannes Eurich / Heinz Schmidt (Hg.), Diakonik. Grundlagen Konzeptionen - Diskurse, Göttingen 2016, 77-109 (87).

8 Thomas K. Kuhn, Werke der Barmherzigkeit. Zu den Anfängen der Diakonie in der Frühen Neuzeit, in: Matthias Freudenberg (Hg.), Diakonie im reformierten Protestantismus, Neukirchen 2018, 19-44 (36-43). 


\section{Diakonie als helfendes Handeln im Kontext des Wirkens Ulrich Zwinglis vor 500 Jahren}

Diakonie bezeichne ich als helfendes, solidarisches Handeln, das aus der jüdisch-christlichen Perspektive begründet oder motiviert wird. Menschen bezeichnen ihr solidarisches Engagement innerhalb und ausserhalb von institutionellen kirchlichen Strukturen und diakonischen Werken nach wie vor mit diesem Begriff. Dabei gehen Heinz Rüegger und ich von folgenden vier grundlegenden Voraussetzungen aus: (1) Die Sache, das Phänomen des helfenden Handelns, nicht der griechische Begriff «diakonein» an und für sich steht im Zentrum der Reflexion. (2) Die gesamtbiblische Tradition wird in den Blick genommen, indem die für den christlichen Glauben konstitutive Nächstenliebe als Ausdruck von Gottes Liebe seinen Geschöpfen, unabhängig von Kultur und Religion, gegenüber theologisch auf die Schöpfungsdimension hin entfaltet wird. (3) Wir gehen von den anthropologischen Einsichten des allgemein menschlichen Helfens aus und (4) erachten die kontextuelle Differenzerfahrung helfenden Handelns in Ländern und Kontinenten als prägender Faktor für die Reflexion christlich konnotierter Praxis helfenden Handelns. ${ }^{9}$ Ausgehend von dieser nicht zufällig im Schweizerischen Kontext gewonnenen Einsicht sollen jetzt Facetten der reformatorischen Leistungen im Bereich der Diakonie beleuchtet werden.

\subsection{Aspekte des Pbänomens Armut zu Beginn des 16. Jabrhunderts}

Armut ist mit Blick auf die Diffusität des lateinischen Begriffes «pauper» ein vielschichtiges Phänomen zu Beginn der reformatorischen Aufbrüche, das nach Thomas Kuhn allgemein mit einem «Mangel» beschrieben

\footnotetext{
9 Vgl. Rüegger / Sigrist, Diakonie (Anm. 4), 29-41.
}

werden kann. ${ }^{10}$ Die heutigen Armutsdebatten mit ihrem Fokus auf die gesellschaftliche Teilhabe von benachteiligten und in unterschiedlicher Weise «Mangel» erleidenden Menschen sind so nicht auf das 16 Jahrhundert zu übertragen. ${ }^{11}$ Folgende grobe Linien skizzieren das Phänomen zu Beginn des 16. Jahrhunderts: Zwei Drittel der städtischen Bevölkerung waren von Armut im Sinne von Existenzgefährdung (primäre Armut) sowie im Sinne von Ausschluss vom öffentlichen Leben (sekundäre Armut) betroffen. Jeder fünfte Einwohner / jede fünfte Einwohnerin verfügte über kein gesichertes Einkommen und war chronisch unterernährt. Ernährungskrisen, hervorgerufen durch klimatische Kälteeinbrüche (1550-1650 galt als «kleine Eiszeit»), die Pest, Kriege, Seuchen und Epidemien sowie Geldmanipulationen der Machtträger mit Wucherzinsen und Geldentwertungen machten der Unterschicht besonders zu schaffen. Armut, Bettelei und Vagantentum wurden als Landplage erlebt, die jedoch im ausgehenden 15. Jahrhundert ihre selbstverständliche, Gott gegebene Akzeptanz verloren. Aufgrund der frühbürgerlichen Tugenden wie Fleiss und Ordnung, Mässigkeit und Disziplin wurden erstmals Kontrollen eingeführt, Bettelzeichen waren üblich geworden. Stigmatisierung von Armut führte einerseits zu Scham und Beschämung Armutsbetroffener, anderseits zur sichtbaren Berechtigung von Unterstützung und Hilfe. ${ }^{12}$

Vorreformatorisch wurde die Fürsorge gegenüber Armen durch Klöster, städtisch gegliederte religiöse Genossenschaften und individuelle

10 Thomas K. Kuhn, Armut als Herausforderung, in: Johannes Eurich u.a. (Hg.), Kirchen aktiv gegen Armut und Ausgrenzung, Stuttgart 2011, 69-95 (81).

11 Vol. dazu mit Blick auf die pflegenden Angehörige von hochbetagten Menschen die Teilhabe-Diskussion bei Simon Hofstetter, Das Unsichtbare sichtbar machen. Pflegende Angehörige und der Auftrag der Diakonie, Zürich 2016, 171-278.

12 Vgl. Kuhn, Werke der Barmherzigkeit (Anm. 8), 27-29. 
Almosengabe organisiert. ${ }^{13}$ Die Trennung zwischen Armut als mönchisches Ideal des freiwilligen, vollkommenen Christen und dem Phänomen der Verarmung breiter Bevölkerungsschichten ist nach Alberto Bondolfi schon im Vorfeld der Reformation vollzogen. ${ }^{14}$ Die sozialpolitischen Aufbrüche verfolgten gemäss Kuhn drei Ziele: «Es ging erstens um die rechtlichen Differenzierungen der Bettler und zweitens um die Bedürftigkeitskontrolle sowie um den Ausbau entsprechender Institutionen. Schliesslich kam drittens auf der moralischen Ebene die Unterscheidung von «würdigen` und «unwürdigen Bedürftigen ins Spiel. Diese Vorsätze städtischer Sozialpolitik wurden in den meisten Städten, unabhängig ihrer Konfession, verfolgt..» ${ }^{15}$ Nach Kuhn ging es in den belegten $\mathrm{Ar}-$ menordnungen der europäischen Städte aus dem späten 15. und dem frühen 16. Jahrhundert um die Kommunalisierung, Rationalisierung, Bürokratisierung wie auch Pädagogisierung der armutsbetroffenen Bevölkerung. «Dazu trat - vermehrt seit den 1520er Jahren - bei der Almosenvergabe das Individualisierungsprinzip, das sich strikt an der regelmässig und streng kontrollierten Bedürftigkeit des Einzelnen orientierte.. ${ }^{16}$ Armutsbekämpfung geschah durch einen ausgebildeten Bildungsanspruch: Bestandene Katechetik-Prüfungen gewährten den Zugang zur Unterstützung, durchgefallene Prüflinge hatten den Entzug der Bettelzeichen zur Folge.

Zusammenfassend lässt sich mit Kuhn beim Blick auf die Armut und die Armutsbekämpfung feststellen, «dass die grundlegenden reformeri-

13 Vgl. Kaspar Von Greyerz, XIV. Die Schweizer Gesellschaft: Familie, Geschlechterrollen und die Armen, in: Amy Nelson Burnett / Emidio Campi (Hg.), Die Schweizer Reformation, Zürich 2017, 549-572 (566).

14 Alberto Bondolfi, Die Debatte um die Reform der Armenpflege im Europa des 16. Jahrhunderts, in: Strohm / Klein (Hg.), Entstehung (Anm. 6), 108.

15 Kuhn, Armut (Anm. 10), 83

16 Kuhn, Werke der Barmherzigkeit (Anm. 8), 31. schen Massnahmen zur Optimierung und Reorganisation der Armenpflege vorreformatorischen Ursprungs sind [...]. Deshalb ist die Gestaltung der frühneuzeitlichen Armenfürsorge nicht ursächlich auf die Reformation zurückzuführen; diese brachte diesbezüglich nichts wesentlich Neues.» ${ }^{17}$ Diese Entzauberung der Exklusivität des reformatorischen Erbes zur Entstehung einer neuen Sozialordnung in Europa ist in der Forschung heute vollzogen. Auch die katholische Kirche, der Humanismus und die Renaissance haben mit ihren Aufbrüchen den Bruch mit den mittelalterlichen Gesellschaftsstrukturen vollzogen. ${ }^{18}$ Angesichts der Götterdämmerung reformierter Exklusivität gilt es jedoch mit Kuhn festzuhalten: «Allerdings intensivierten die reformatorischen Bewegungen aufgrund ihres inhärenten sozialen Engagements nicht nur die armenfürsorgerischen Diskurse, sondern auch die praktische Umsetzung. $)^{19}$ Diese praktische Umsetzung hat einen theologischen und einen gesellschaftspolitischen Fokus, die ich am Beispiel der Umwälzungen in Zürich darstellen möchte.

\subsection{Biblisch-theologische Einblicke}

Heinrich Bullinger (1504-1575), Zwinglis Nachfolger, beschreibt in seiner Reformationsgeschichte kurz vor seinem Tod 1573 Zwinglis Wirken in Zürich und fasst auf den ersten Seiten grossartig seine homiletischtheologische wie auch politische Überzeugungskraft zusammen. Ein grosses «Gläuf» von allerlei Menschen sei von Anbeginn seiner Predigttä-

17 Kuhn, Werke der Barmherzigkeit (Anm. 8), 35. Vgl. auch ders., Armut (Anm. 10), 84

18 Vgl. dazu Robert Jütte, Tendenzen öffentlicher Armenpflege in der Frühen Neuzeit Europas und ihrer weiter wirkenden Folgen, in: Strohm / Klein (Hg.), Entstehung (Anm. 6), 78-104 (81); Sozialwissenschaftliches Institut der EKD (Hg.), Diakonie (Anm. 3), 18; Gottfried Hammann, Die Geschichte der christlichen Diakonie, Göttingen 2003, 174 .

19 Kuhn, Werke der Barmherzigkeit (Anm. 8), 35. 
tigkeit da gewesen. Er habe Gott als den Vater gepriesen und gelehrt, dass die Menschen auf Gottes Sohn, Jesus Christus, auf den Heiland vertrauen sollten. «Die buss oder besserung des läbens, vnd Christlich lieb vnd trüw, teyb er häfftig. Die laster, alls der Müssigang, unmaass in ässen trincken kleydern, frässerey vnd füllery, vndertrucken der Armen, pensionen vnd kriegen strafft er ruch, trang ernstlich vff das ein oberkeit gricht vnd rächt hiellten, wittwen, vnd weysen schirmpten, vnd das man die Eydgenossische fryheit sich zu behalten flysse, der fürsten und Herren bulen vssschlüge.. $\rangle^{20}$

Ich teile Kuhns Meinung: «Von Anfang an dürfte sich Zwingli demnach in Zürich sozialfürsorgerisch und diakonisch engagiert und damit Kontroversen und weiterführende soziale Überlegungen in der Stadt ausgelöst haben». Diese Einsicht kann biografisch am Beispiel seines Verhaltens während der Pestepidemie im Herbst 1519 erhellt werden, wo er nach wenigen Wochen Kuraufenthalt in Bad Pfäffers wieder als Leutpriester, Pfarrer des Volkes, nach Zürich zog, um für die Kranken und Sterbenden dazu sein. Er erkrankte selber und schrieb nach der Genesung sein berühmtes Pestlied. ${ }^{21}$

Für unsere Fragestellung interessanter sind jedoch die theologischen Grundlinien Zwinglis, die in Heinrich Bullingers zusammenfassender Predigttätigkeit aufscheint: Wenn es um die Bekämpfung der «vnderdrucken der Armen» geht, dann spiegelt sich das Verhalten der Glaubenden im Verhalten Jesu Christi. Denn Christus hat nicht über die Armen geschwätzt, sondern hat sich mit ihnen solidarisch gezeigt. Die Nachfolge

20 Heinrich Bullinger, Reformationsgeschichte nach dem Autographen, herausgegeben auf Veranlassung der vaterländisch-historischen Gesellschaft in Zürich von J.J. Hottinger und H.H. Vögeli, Frauenfeld 1838, 12f.

21 Vgl. zum biografischen Kontext des Pestliedes: Christoph Sigrist, Anna Reinhart und Ulrich Zwingli. Von der Tochter eines Gastwirts zur Frau des Reformators, Freiburg im Breisgau 2017, 89-95.
Christi zeigt sich in der Gleichförmigkeit seines Wandels: «Ein Christ syn ist nit schwätzen von Christo, sunder wandlen, wie er gewandelt hat. Ein Christ syn ist der schönst und zierlichest Adel, der in dem Himmel und uff erden sin mag.» ${ }^{22}$ Angesichts seiner Erfahrung als Pfarrer in der Armee wenig erstaunlich, malt Zwingli oft diese Nachfolge im Bild des Hauptmanns Christus: «Sehe ein yeder uff sinen Houptmann Christum Jesum; der wirt uns nit verführen..»3 Nach Gottfried Locher bewirkt dieses Bild aus Zwinglis Text zweierlei: «Äusserlich durch die Vermahnung zur Standhaftigkeit in der Lehre oder im Leiden, inwendig «durch seinen Gayst»» ${ }^{24}$. Christi Geist ist jedoch Gottes Geist und die Wirkung dieses Geistes ist, den Menschen zu Gott selber zu ziehen. «Sobald du das gloubst, wiß dich ietz gezogen sin von dem vatter, und das du meinst din arbeit sin, ist des geist gottes, der heimlich in dir würckt. Denn niemans kumpt zuo mir, spricht Christus Joannis am 6. [Joh. 6. 44], es zühe inn dann min vatter, der in den himlen ist.» ${ }^{25}$ Dieser Zug zum Vater zieht sich wie «ein roter Faden» ${ }^{26}$ durch sein theologisches Nachdenken. Er knüpft diesen Faden mit dem Gedanken des Bundes, den Gott durch seine Geschichte mit dem Volk Israel (Altes Testament) und durch Jesus Christus (Neues Testament) mit den Menschen geschlossen hat und dessen inhaltlicher Kern das Gute in, durch und mit Gott ist. ${ }^{27}$ Dieses schöpferische, überströmende Gute drängt dazu, ausgeteilt zu werden und zeigt sich nach Zwingli beispielhaft in der liebenden $\mathrm{Zu}$ -

22 Zwingli, Sämtliche Werke 3 (Anm. 1), 407.

23 Zwingli, Sämtliche Werke 5 (Anm. 1), 307.

24 Gottfried Locher, Die Theologie Huldrich Zwinglis im Lichte seiner Christologie, Zürich 1952, 19.

25 Huldreich Zwingli, Sämtliche Werke I (Anm. 1), 374.

26 Locher, Theologie (Anm. 24), 49

27 Vgl. zur Bundestheologie: Emidio Campi, XI. Das theologische Profil, in: Burnett / ders. (Hg.), Die Schweizer Reformation (Anm. 13), 449-493 (482-491). 
wendung Christi bei der Fusswaschung. ${ }^{28}$ Wandeln wie Christus gewandelt ist, heisst, das Gute der Schöpfung als Güte des Bundes Gottes mit den Menschen als Gut des helfenden Handelns der Diakonie den Armen zu verschenken. So sind wir Geschirr Gottes, ${ }^{29}$ sein Hafen, sein Gefäss. 30

Diese «überströmende» Armutsbekämpfung führt zu fünf grundlegenden Entscheidungen: Die Abkehr von Buhlen der Fürsten und Bischöfe mit ihrer Gier, ${ }^{31}$ die Umlenkung der Gelder weg von den Altären drinnen in der Kirche hin zu den Armen draussen vor der Tür, ${ }^{32}$ die grundlegende Bedeutung der Freiheit als Verantwortung des einzelnen für das Gemeinwesen, die Bildung als Überwindung vom Müssiggang sowie die Übertragung Aufgabe der «Beschirmung der Armen» zum Staat. Die mittelalterliche Armenpflege - namentlich die Verortung der

28 Vgl. zur ethischen Dimension der theologischen Verortung des Guten bei Gott: Matthias Neugebauer, Ulrich Zwinglis Ethik. Stationen - Grundlagen - Konkretionen, Zürich 2017, 71-89, bes. 85ff.

29 «[...] hoc unum Christum obtestans, ut massculo omnia pectore ferre donet et me, figulinum suum, rumpat aut firmet, ut illi placitum sit.» Zwingli, Sämtliche Werke 7 (Anm. 1), 344.

30 Aus dem Pestlied 1519. «Din haf bin ich. Mach gantz ald brich.» Zwingli, Sämtliche Werke I (Anm. 1), 67.

31 «Ueber ein thierfräsigen wolff stürmpte man und den wolffen, die lüth verderbind, wölle nieman rächt werren. Sy tragind billich rote huet und mäntel; dann schütte man sy, so fallind duggaten und kronen herus; winde man sy, so ründt dines suns, bruoders, vatters und guoten fründts bluot herus.» Zwingli, Sämtliche Werke I (Anm. 1), 73.

32 «Ist es umb des geltes willen ze thuon, so ist es der recht uppig bapstbschiß, damit man die narren umb die müler salbet, das sy gold und gelt gebind, das er damit die mulesel mit syden und gold beschleuffe. Welchs aber wir den dürfftigen bilden gottes, den armen menschen, geben soltend, so henckend wir's an des menschen bildnus; denn die götzen sind bildnussen des menschen, aber der mensch ist ein bildnus gottes.» Zwingli, Sämtliche Werke IV (Anm. 1), 122.
Unterstützung der Armen Busssakrament mit den damit verbundenen Reueleistungen, Sündenbekenntnissen und finanziellen Abgeltungsleistungen $^{33}$ - wurde in Aufnahme der spätmittelalterlichen Kritik am Betteltum ${ }^{34}$ durch die schon in vielen Städten praktizierte politische Verortung abgelöst. Zusammenfassend:

- Die Armut ist ein theologisches Problem, weil Gottes Güte zu dem fliesst, dem Böses widerfahren ist. Gott ist nicht neutral, sondern parteiisch, er nimmt Partei für die Geringen, Waisen, Elenden, Bedürftigen, befreit sie aus der Hand der Frevler (vgl. Psalm 82,3f)

- Die Armut ist ein finanzielles Problem, weil das Geld in sich das soziale Kapital der Armutsbekämpfung in sich trägt.

- Die Armut ist ein politisches Problem dadurch, dass der Staat die Verantwortung trägt.

- Die Armut ist ein pädagogisches Problem, weil durch Bildung eine Form von Armut überwunden wird.

- Die Armut bleibt ein kirchliches Problem, weil Kirchen Räume, Menschen und Kapital dafür einsetzen können und dies mit einem Gottes- und Menschenbild tun, in welchem sich die göttliche Gerechtigkeit in der Aushandlung menschlicher Gerechtigkeit spiegelt.

\subsection{Politische Implikation: Almosenordnung von 1525}

Die in Zürich in den 20er Jahren des 16. Jahrhunderts praktizierte Umsetzung dieser theologischen Grundentscheidung, den Verdienstcharakter der Armutsbekämpfung nicht mehr in der Heilsökonomie, sondern in der gesellschaftspolitischen Ökonomie heftig zu kritisieren, war eine

33 Vgl. dazu: Kuhn, Armut (Anm. 10), 83

34 Vgl. zur Kritik: Bondolfi, Debatte (Anm. 14), 116-120. 
Folge von längerdauernden gesellschaftlichen Transformationen. Erste Impulse und Aufbrüche in der Politik europäischer Städte mit ihren Armenordnungen und sogenannten «Gemeinen Kasten», einer «staatlichen» Armenkasse, wiesen den Weg. ${ }^{35} 1525$ war das entscheidende Jahr, in dem die Reformation der Kirche nach erfolgten Disputationen und Beschlüssen in den beiden Räten zur Transformation der Gesellschaft führte. Die Einsetzung des Ehegerichts, die Emanzipation von kirchlichen Rechtsprechungen, die Gründung der Theologenausbildung (die «Prophezey» im Chor des Grossmünsters), die Einführung des evangelischen Gottesdienstes mit dem Abendmahl sowie die Inkraftsetzung der Almosenordnung bauten den Stadtstaat Zürich und das dazugehörige Umland grundlegend um. Diese soziale Ordnung ist eine bahnbrechende Weiterführung der sozialen Umbrüche, die mit Christian Moser «im Wesentlichen bis zur Helvetik hin das Fundament der institutionellen Armenfürsorge in Zürich bilden sollte.» ${ }^{36}$

Was ist der Inhalt dieser Ordnung? ${ }^{37}$ Zentrales Herzstück war der «Mushafen», angesiedelt am ehemaligen Predigerkloster, wo eine tägliche Essensabgabe eingerichtet wurde, verbunden mit der Abgabe von Kleidern, der Übernahme von Schulden oder kostenfreier Arztbehandlung. Ein vom Rat eingesetzter Obmann und vier Pfleger waren verantwortlich für die anvertrauten Gelder, die im «Gemeinen Kasten» lagen, der nur zusammen mit einem Leutpriester, also auch Zwingli, geöffnet werden konnte. Die Gelder stammten aus fünf Quellen: Aus dem säkulari-

35 Vgl. Kuhn, Werke der Barmherzigkeit (Anm. 8), 30-36; Kuhn, Armut (Anm. 10), 85-88.

36 Christian Moser, Institutionelle Armenfürsorge in Zürich 1520-1600, in: André Holenstein, u.a. (Hg.), Reichtum und Armut in den schweizerischen Republiken des 18. Jahrhunderts, Genf 2010, 33-49 (34).

37 Vgl. zum Text der Almosenordnung in Zürich: Klein, Almosenordnung (Anm. 6), $102-107$. sierten Kirchengut (Klöster), aus Zehntrechten des Grossmünsterstifts, aus Kollekten und Spenden, die im Zusammenhang mit Gottesdiensten eingesammelt wurden, aus dem Verkauf und der Einschmelzung von Sakral- und Ziergeräten sowie schliesslich aus den Überschüssen der wirtschaftlichen Leistungen der ehemaligen Klöster. Ein Laie zusammen mit einem Pfarrer hatte zudem den Auftrag, in aufsuchender sozialer Arbeit in den sieben Stadtkreisen (Wachten) Hausbesuche und Unterstützungen zu leisten, die in Büchern vermerkt wurden. Arme wurden unterstützt, die unverschuldet durch Krieg, Feuer, Teuerung, Krankheit, Kinderreichtum und hohes Alter in Not gerieten. Keine Unterstützung bekamen faule, in Luxus schwelgende Personen, die dem Kirchgang fernblieben, prassten oder Fremde waren. Die Empfänger von Almosen mussten ein metallenes Zeichen tragen, ausnahmen waren «Ehrenleute». Bettelei wurde mit acht Tagen Almosenverweigerung bestraft. Fremde Bettler wurden für eine Nacht aufgenommen, um sie dann für ein halbes Jahr ausserhalb der Stadtmauern zu schicken. Bei Missachtung des Verbots drohte Gefängnisstrafe. Fremde wurden dahin zurückgeschickt, woher sie kamen.

Was ist der Charakter dieser Ordnung? Nach Christian Moser sind sechs Aspekte auszumachen: Erstens besteht eine Verschränkung von Freisinn und Gemeinsinn, d.h. von individueller Verantwortung des Einzelnen und solidarischer Verpflichtung des Gemeinwesens; zweitens erfolgt eine Verschiebung der Fürsorge aus dem kirchlichen in den staatlichen Kompetenzbereich; drittens wird durch die Notwendigkeit der moralischen und sittlichen Vorleistungen für den Empfang der Hilfeleistung eine Disziplinierung eingeführt; viertens ergibt sich daraus eine grosse Deckungsgleichheit von Kirche und Staat als die eine Res publica christiana; fünftens erfolgt damit in theologischer Perspektive eine Ablösung vom heilsrelevanten Handeln an den Armen zur bürgerlichen 
Christenpflicht mit den Armen; sechstens ist auf die katalysatorische Wirkung der reformatorischen Kräfte im Bereich der Fürsorge hinzuweisen. 38

Wie stand es mit der Diakonie als christlich begründetes helfendes Handeln? Die Einsetzung von nicht ordinierten Pflegern ist in dieser Art einmalig und zeigt erste Linien zu einem allgemein, im Menschsein selber liegenden Phänomen von Armut und Hilfe. Mit Kuhn ist festzuhalten «Diakonie erscheint bei Zwingli demnach als weltlicher Sozialdienst». Dieser weltliche Sozialdienst ist für Zwingli selbstverständlich Ausfluss der Güte Gottes, des Wortes Gottes selber und setzt den Pfleger zwischen dem Armen und dem Pfarrer auf eine Linie, ohne die Ordination zum kirchlich eingegliederten Diakon zu fordern. In seinen Schlussreden schreibt Zwingli 1523: «Also laß ich hie gern priester sin, die by der kilchen lerend, die, so das gotswort verkündend, die, so die griechisch und hebraisch sprach tollmetschend, die predgend, die artznend, die die krancken heimsuochen, die, die hilff und almuosen den armen zuoteilend, die spysend; denn dise stuck ghörend alle zuo dem wort gottes.» ${ }^{39}$ Ich gehe mit Kuhn einig: «Bei Zwingli wird erkennbar, dass derjenige, der sich mit Diakonie im karitativen Sinn beschäftigt, auch Diener des Wortes, der evangelischen Botschaft ist, ohne jedoch im eigentlichen und engeren Sinn Prediger zu sein.» ${ }^{40}$

Doch in Zürich blieb es dabei: Diakone wurden zu Sozialarbeitern, sie hatten keine im engeren Sinne kirchliche Funktion mehr. Mit Kuhn

38 Vgl. Moser, Armenfürsorge (Anm. 36), 38-42.

39 Zwingli, Sämtliche Werke 2 (Anm. 1), 441.

40 Kuhn, Werke der Barmherzigkeit (Anm. 8), 41. Gottfried Hammann unterstreich diesen Sachverhalt in Zürich: «Wenn beide Priester (Pastoren und Diakonie; erg. CS) waren, d.h. ordinierte Diener des Wortes, so verkünden die einen die biblische Botschaft mit Worten, die anderen mit Taten, ohne dass die beiden Dienste notwendigerweise das Privileg eines einzigen besonderen Amtes gewesen wären.» Hammann, Geschichte (Anm. 18), 228 ist festzuhalten: «In der im Jahre 1525 erlassenen Kirchenordnung war folgerichtig das eigentliche kirchliche Diakonat abgeschafft.» ${ }^{41}$ Hörnig ist beizupflichten, wenn er kurz und bündig festhält: »Huldrich Zwinglis (1484-1531) Theologie war deutlich sozialethischer akzentuiert als die Luthers.» ${ }^{42}$ Dies war in Zürich so, jedoch nicht in der ganzen Eidgenossenschaft.

\subsection{Kontextuelle Färbungen}

Mit dieser Einsicht wird der Blick auf drei Seiten hin geöffnet.

Einerseits zur Diskrepanz von Theorie und Praxis: In Bezug auf die Umsetzung der diakonischen Aufbrüche gilt es mit Kuhn schlicht festzuhalten: «Die Differenzen zwischen Theorie und Praxis reformatorischer Ekklesiologie sind offensichtlich [...]. Es war wohl das Ziel der Reformation, die besondere kirchliche Bedeutung der christlichen Diakonie wieder zur Geltung zu bringen. Trotz einiger Erfolge bei der Optimierung der Armenfürsorge gelang es ihr jedoch aufs Ganze nicht, den Diakonat als ekklesiales Amt nach dem Vorbild der Ur- und Alten Kirche in der Praxis wiederherzustellen.» ${ }^{43}$ Und Hammann konstatiert, «dass der reformierte Diakonat - ausser bei Zwingli - grösstenteils blosse Theorie blieb. Er wurde von der jeweiligen Ämtertheologie überlagert und ausserdem durch die Zurückhaltung und den Widerstand der weltlichen Obrigkeit an seiner erfolgreichen Realisierung seit Beginn der Reformation behindert. ${ }^{44}$

Zweitens zur Bildung und Arbeit. In Zürich konnte die Pfarrschaft unter Heinrich Bullinger, Zwinglis Nachfolger, mit sogenannten «Fürträ-

41 Kuhn, Werke der Barmherzigkeit (Anm. 8), 42.

42 Hörnig, Geschichte (Anm. 7), 87.

43 Kuhn, Werke der Barmherzigkeit (Anm. 8), 43.

44 Hammann, Geschichte (Anm. 18), 296f. 
gen» beim Rat zur Frage der Armutsbekämpfung Einfluss nehmen. In einem Vorstoss vom 23. März 1558 legten die Pfarrer dem Rat ans Herz, die Pflicht zur Fürsorge nicht zu vernachlässigen. Anderseits sollten die Missstände aufgehoben werden beim Rechnungswesen, dem Einzug von Almosengeldern, dem missbräuchlichen Bezug von Armengeldern und der Duldung von Bettlern, die nicht arbeiteten, sondern einfach «schamlos herumlaufen». ${ }^{45}$ Damit wollten sie der Kritik der katholischen Kirche begegnen, der Staat würde ihren Armen weniger Unterstützung zukommen lassen. Im Sommer 1572 forderte Bullinger mit seinen Kollegen noch weiterführende Massnahmen: Almosenempfangenden soll das Stimmrecht bei Gemeindeversammlungen entzogen werden, der Besuch in Wirtshäusern untersagt sowie die Pflichtarbeit insbesondere im Textilbereich eingeführt werden. ${ }^{46}$ Wenn die «schamlos herumlaufenden bettler» durch Bildungsmassnahmen aus der Armut geholt werden sollten, so stellt dies eine Vorform des heutigen Prinzips der Hilfe zur Selbsthilfe dar; dieses wurde in Genf bereits von Johannes Calvin forciert. Frank Jehle bringt es in seinen Ausführungen zu Wirtschaft und Reformation auf den Punkt: «Calvin regte in Genf staatliche Arbeitsbeschaffungsmassnahmen und - noch weitsichtiger! - Berufsbildungsprogramme für Arbeitslose an [...]. Gut geschulte Arbeiter produzierten mehr. Und mit ihrem Lohn zufriedene Angestellte ebenfalls! Dazu kommt, dass Calvin sich offensichtlich mehr als andere Theologen für die Wirtschaft interessierte.» ${ }^{47}$ Durch Bildung und damit durch Arbeit wird Armut bekämpft! Ohne auf die sogenannte Weber-These vertieft einzugehen, ${ }^{48}$ kann auf-

45 Moser, Armenfürsorge (Anm. 36), 43.

46 Moser, Armenfürsorge (Anm. 36), 44.

47 Frank Jehle, Du darfst kein riesiges Maul sein, das alles gierig in sich hineinfrisst und verschlingt. Johannes Calvin, Basel 1996, 81.

48 Vgl. zur bekannten These Max Webers, wonach der Calvinismus des Ende 16. und 17. Jahrhunderts einen grossen Einfluss auf die Arbeitsethik und kapitalistische Kul- grund der angeführten Belege durchaus festgehalten werden, dass ein konstitutiver Beitrag der Reformierten zur diakonischen Praxis im Engagement zur Armutsbekämpfung in diesen Bildungs- und Arbeitsprogrammen liegt. Kaspar von Greyerz redet in diesem Zusammenhang von der «Gewichtung der Christianisierung und Heiligung der Gemeinschaft bei den oberdeutschen und Schweizer Reformatoren.» ${ }^{49}$

Drittens zum föderalistischen Konzept der Eidgenossenschaft. Was in Zürich galt, war so in Genf nicht zwingend gültig. Gottfried Hammann legt in seiner gross angelegten Untersuchung der reformierten Situation in der Schweiz das Bild des reformatorischen Scheiterns des Diakonats differenziert aus. ${ }^{50}$ In Zürich wurde der kirchlich eingegliederte Diakon zum vom Staat angestellten, nicht ordinierten Sozialarbeiter. Durch den Prozess der immer stärkeren Angleichung zwischen Pastoren und dem Seelsorge- und Diakonieamt in der unmittelbaren weiteren Entwicklung ist es verständlich, dass bei Heinrich Bullinger, dem Nachfolger Zwinglis, die liturgische Einbindung des diakonischen Dienstes wieder aufgegriffen wurde - eine «erstaunliche Rehabilitation des streng kirchlichen Diakonieamtes.» ${ }^{51}$ Nach Bullinger setzte sich in Zürich die Exklusivität des Wortdieners im engen Sinne des kirchlichen Amtes mit seiner Ordination durch. ${ }^{52}$ Bei Calvin in Genf wurde - in Aufnahme der Ämtertheolo-

tur ausübte: Jehle, Du darfst kein riesiges Maul sein (Anm. 47), 11-27; Marco Hofheinz, «Die Gläubigen hatten alles gemeinsam». Die Gütergemeinschaft der Urgemeinde nach Johannes Calvin. Zugleich ein Beitrag zur sog. Weber-These, in: Matthias Freudenberg u.a. (Hg.), Diakonie im reformierten Protestantismus, Neukirchen 2018, 45-57.

49 Von Greyerz, Die Schweizer Gesellschaft (Anm. 13), 563

50 Hammann, Geschichte (Anm. 18), 295-298.

51 Hammann, Geschichte (Anm. 18), 228

52 Darin sehe ich auch den Grund, dass aktuell in der Evang.ref. Kirche des Kantons Zürich die mit dem Wort «Beauftragung» erfolgte Aufnahme der Sozial-Diakoninnen und Sozial-Diakone getrennt von der Ordination der Pfarrpersonen erfolgt, im Un- 
gie Martin Bucers in Strassburg - die Lehre von den vier Ämtern weiterentwickelt, die dann vielfach aus Deutscher Perspektive als einziger spezifisch reformierter Beitrag zur diakonischen Situation in der Schweiz genannt wird. ${ }^{53}$ Dies ist jedoch schlicht falsch, wie nur die wenigen Hinweise eindrücklich aufzeigen. Es ist das Verdienst von Martin Sallmann mit Blick auf die Diakonie in der Schweiz des 19. Jahrhunderts den Sachverhalt festzuhalten, der seit Beginn der Schweizerischen Reformation in den unterschiedlichen Kantonen für die Diakonie bis in die Neuzeit gilt: «Im 19. Jahrhundert war das diakonische Handeln der Reformierten in der Schweiz sowohl institutionell als auch gesellschaftlich breit angelegt. Diese Vielfalt gehört zur Diakonie im reformierten Protestantismus> der Schweiz.. ${ }^{54}$

\section{Wirkungen des reformatorischen Erbes}

Kaspar von Greyerz nennt drei konstitutive Aspekte für die Wohlfahrtspflege im modernen Sozialstaat: «Erstens staatliche Unterstützung für Kranke, Arbeitslose und Arme; zweitens ein staatliches Rentensystem und eine Steuerpolitik, die für ein gewisses soziales Gleichgewicht sorgt; drittens die Aufrechterhaltung von Sicherheit im öffentlichen Raum.» ${ }^{55}$ Für ihn wirken reformatorische Aufbrüche im Bildungswesen und Schulen, Katechismusunterricht bei Kindern und Jugendlichen, Streichung

terschied zum Beispiel zur der Evang.-ref. Kirche des Kantons St. Gallen, wo die unterschiedlichen Dienste miteinander in einer Ordinationsfeier in den Dienst der Kirche aufgenommen werden.

53 Jüngst in frappant deutlicher Art und Weise: Gaus, Reformation (Anm. 5), $261 \mathrm{f}$.

54 Martin Sallmann, Diakonie in der Schweiz des 19. Jahrhunderts, in: Matthias Freudenberg u.a. (Hg.), Diakonie im reformierten Protestantismus, Neukirchen 2018, 125-139 (139).

55 Von Greyerz, Die Schweizer Gesellschaft (Anm. 13), 570. von Heiligentagen und Festen, Unterbindung von örtlichen Kirchweihfesten wie auch die Ausgestaltung der Armenordnungen in Sittenzuch und sozialer Disziplinierung in die Entwicklung der moderne Wohlfahrtspflege ein. ${ }^{56}$ Wie unterschiedlich diese Wohlfahrt im Bereich der Pflege von Angehörigen in den Kantonen der Schweiz organisiert wird, geschweige denn im europäischen Vergleich, zeigt Simon Hofstetter eindrücklich in seiner Studie auf..$^{57}$

Alberto Bondolfi unterscheidet drei Parallelen zwischen dem 16. und dem 21. Jahrhundert, die für die Diakonie als helfendes Handeln konstitutiv sind: In Bezug auf die gesellschaftliche Struktur sind Prozesse von Marginalisierungen wie Arbeitslosigkeit und Vagabundentum in beiden Jahrhunderten feststellbar. Zugleich sind die Massnahmen zur Bewältigung dieser Probleme «frappant» wie zum Beispiel, dass Arme zu ihren Heimatorten zurückkehren sollen. Schliesslich ist den jeweiligen gesellschaftlichen Diskurs die Tendenz gemeinsam, «Kontrolle und Repression den Ausgegrenzten gegenüber legitimieren zu wollen.» ${ }^{58}$

Ich versuche zum Schluss unter Aufnahme der strukturellen Voraussetzungen helfenden Handelns aufgrund meiner Erfahrung in der Praxis in wenigen Strichen die für mich einsichtigen Aspekte der schweizerischen - auf Zürich fokussierten - reformatorischen Aufbrüche vor 500 Jahren zu skizzieren. Dabei werden die spezifisch schweizerischen Entwicklungslinien, namentlich Pietismus, Aufklärung, Erweckungsbewegung im 19. Jahrhundert sowie religiöser Sozialismus und kirchliche Diakonie im 20. Jahrhundert, nicht berücksichtigt. Die präzise Aufarbeitung gerade der neueren Diakoniegeschichte in der Schweiz ist ein Forschungsschwerpunkt der Dozentur für Diakoniewissenschaft an der theologischen Fakultät der Universität Bern in den nächsten fünf Jahren.

56 Von Greyerz, Die Schweizer Gesellschaft (Anm. 13), 571f.

57 Vgl. Hofstetter, Das Unsichtbare sichtbar machen (Anm. 11), 87-162.

58 Bondolfi, Debatte (Anm. 14), 145 
Ein typisch schweizerisches Phänomen ist die Übertragung der sozialen Verantwortung der Wohlfahrt und Bekämpfung der Armut an den Staat und verschiedene gesellschaftliche Akteure. Mit der Sache der Diakonie machen Bund, Kantone und Gemeinden Staat, nicht die Kirchen. Im Unterschied zu Deutschland hat sich in der Schweiz im 19. und 20. Jahrhundert kein grosses diakonisches Werk herausgebildet. Die ökonomisch intensiven und marktförmigen Leistungen im Bereich von Arbeit und Existenzsicherung, Gesundheit und Wohlergehen, Zugehörigkeit und Teilhabe haben die staatlichen Behörden zu leisten. Das für das kirchliche Leben konstitutiv diakonisch helfende Handeln wird lokal erfasst und kirchlich wie institutionell verankert. Diese typisch schweizerische Subsidiarität kirchlichen Engagements gegenüber dem Staat hat in den letzten beiden Jahrzehnten eine Klärung des inhaltlichen Auftrages der diakonischen Leistung von Kirchgemeinden, gesamtkirchlichen Diensten, diakonischen Werken und kirchlichen Hilfswerken vonnöten gemacht. Die sogenannte «diakonische Zwölffeldertafel» des Diakoniekonzeptes der evang.-ref. Kirche des Kantons Zürich ist ein gutes Beispiel dafür. ${ }^{59}$

In biblisch-theologischer Perspektive hat der mit Heinz Rüegger entwickelte schöpfungstheologische Ansatz im deutschsprachigen Raum kontroverse und zum Teil hitzige Diskussionen ausgelöst. Angesichts der unterschiedlichen theologischen Gewichtungen der Reformatoren auch in der Schweiz wird auch in Zukunft einerseits die theologische Begründung helfenden Handelns für das kirchliche Leben und das diakonische Wirken konstitutiv sein. Die Multiperspektivität theologischer Ansätze gewinnt jedoch angesichts der pluralen Gesellschaft immer mehr an Gewicht. Die theologische Verarbeitung der Diakonie ist angesichts der interkulturellen Zusammensetzung bei helfenden und pflegenden Beru-

59 Vgl. dazu: Frieder Furler, Diakonie - eine praktische Perspektive. Vom Wesensmerkmal zum sichtbaren Zeichen der Kirche, Zürich 2012, 93-95. fen zu einer notwendigen Arbeit in der Diakoniewissenschaft wie auch im praktischen Vollzug vor Ort geworden. Erste Ansätze und Impulse sind erfolgt. ${ }^{60}$ Die vertiefte Auseinandersetzung, wie die von den Reformatoren und den Gründungsvätern und -müttern der diakonischen Aufbrüche im 19. Jahrhundert forcierte christologische Begründung (mit ihrem Fokus auf die Rechtfertigung des Gottlosen allein aus Glauben) mit anderen theologischen Zugängen in eine zielführende neue Sichtweise weiterentwickelt werden kann - diese Auseinandersetzung muss in Zukunft noch viel gründlicher geführt werden.

Da helfendes Handeln zum «prosozialen Naturell» 61 des Menschen gehört, also anthropologisch im Humanum eingelagert ist, genauso wie das asoziale Naturell von Fressen und Gefressen werden, liegt es in dieser Einsicht nahe, die Wohlfahrt als «Welfare-Mix» ${ }^{62}$ oder «Wohlfahrtspluralismus» ${ }^{63} \mathrm{zu}$ verstehen. Die Almosenordnung in Zürich beschreibt erste Ansätze eines solchen Zusammenspiels relevanter Player im Bereich der Bekämpfung der Armut. Daraus ergeben sich folgende Herausforderungen für das diakonische Handeln in der Schweiz: Erstens die ökonomische Erfassung von diakonischen Leistungen in Kirchen, zweitens die inhaltliche Beschreibung des diakonischen Auftrages, wie er in den Diakoniekonzepten der 10er Jahre dieses Jahrhunderts in den einzelnen Kantonen entfaltet wurde, drittens die Reorganisation der diakonischen Organisationen und Strukturen mit der Wirkung ins Inland und Ausland, wie sie jüngst durch die Plattform «Diakonie Schweiz» ${ }^{64}$ erfolgt ist, vier-

60 Vgl. dazu: Sigrist / Rüegger (Hg.), Helfendes Handeln (Anm. 4).

61 Sarah Blaffer Hrdy, Mütter und andere. Wie die Evolution uns zu sozialen Wesen macht, Berlin 2010, 48.

62 Rüegger / Sigrist, Diakonie (Anm. 4), 246.

63 Hofstetter, Das Unsichtbare sichtbar machen (Anm. 11), 82.

64 Vgl. zur Diakonie Schweiz, URL: https://www.diakonie.ch/, abgerufen am 16.2 .2018 
tens die Förderung der Kooperationen unterschiedlicher Organisationen, Kirchgemeinden, Pfarreien, Gruppierungen und Initiativen in Stadtteilen, Quartieren und urbanen sozialen Räumen, um das Zusammenleben, Zusammenwohnen und Zusammenarbeiten intergenerativ und auf spezifische Milieus ausgereichtet zu unterstützen. Diese neuen Formen diakonischen Arbeitens werden in den einzelnen Kirchgemeinden und diakonische Werken sichtbar und seit ein paar Jahren unter dem Claim «Urbane Diakonie» zusammengefasst und gefördert. ${ }^{65}$

Der gesellschaftliche Kontext ist nicht nur zwischen der Schweiz, Deutschland und dem übrigen Europa, sondern auch zwischen den einzelnen Kantonen und Regionen der Schweiz unterschiedlich. Die Gesellschaft zur Reformationszeit war ein durch und durch christlich geprägtes Gemeinwesen. Heute leben wir in einem durch und durch plural gewordenen gesellschaftlichem Umfeld. Nicht mehr die Unterscheidung zwischen altem und neuem Glauben, die Frage nach der reformierten und katholischen Identität, ist die zentrale Herausforderung. Die Entwicklung von pluralen religiösen Gemeinschaften, Verhaltensmustern, Lebens- und Glaubensformen lassen nach der Forschungsgruppe unter Leitung des Religionssoziologen Jörg Stolz aktuell vier Gestalten des (Un-) Glaubens in der Schweiz beschreiben: Die institutionelle, alternative, distanzierte und säkulare Form. ${ }^{66}$ Bei dieser Entwicklung steht die Frage nach dem Christsein innerhalb der unterschiedlichen Konzepte von Religion in Theorie und Praxis im Vordergrund. In der Suche nach dem Christsein spielen selbstverständlich konfessionelle Prägungen und konfessionelle Identität stiftende Suchbewegungen eine grosse Rolle. Mit Blick auf die Diakonie habe ich versucht, den entkonfessionalisierten Kontext konstitutiv für das Konzept einer kulturellen Kohärenz helfen-

65 Vgl. dazu: http://www.urbanediakonie.ch/, abgerufen am 16.2.2018.

66 Vgl. Jörg Stolz u.a., Religion und Spiritualität in der Ich-Gesellschaft, Zürich 2014, $65-78$. den Handelns weiterzuentwickeln. ${ }^{67}$ Darunter verstehe ich die konzeptionelle Beschreibung helfenden Handelns unter Berücksichtigung der kulturellen Veränderungen, Brüche und Herausforderungen der Gesellschaft. Unter dieser Perspektive kann die Loslösung der Armutsbekämpfung weg von der Heilsökonomie hin zur gesellschaftspolitischen Ökonomie der «Gemeinen Kaste» Ausdruck einer solchen diakonischen kulturellen Kohärenz verstanden werden. War es vor 500 Jahren die Korruption in der Armutsbekämpfung, ist es heute die Überforderung im Zusammenleben unterschiedlicher Kulturen und Religionen. Im Bereich der Seelsorge als Teilaspekt helfenden Handelns und so in der Schnittmenge zur Diakonie angesiedelt, kann die jüngst erschienene Handreichung für die christliche Seelsorge mit dem Titel «Christlich-muslimische Trauerfälle» als Ausdruck dieser kulturellen Kohärenz angeführt werden. ${ }^{68}$ Die reformierten Kirchen Bern-Jura-Solothurn, die katholische Kirche Region Bern und die Christkatholische Kirche des Kantons Bern haben diese Handreichung gemeinsam erarbeitet. Angesichts der aktuell 346000 Musliminnen und Muslime in der Schweiz, das sind rund 5\% der gesamten Bevölkerung, ist nicht mehr der heilsökonomische Gebrauch und Missbrauch der Kirche gegenüber den Armen, sondern die seelsorgerliche Begleitung der muslimischen Bevölkerung mit Blick auf Sterben und Tod die kulturelle Herausforderung, die nach einem kohärenten helfenden Handeln fragt. «Bei Trauerfällen, in denen christliche und muslimische Personen involviert sind, ist eine religionssensible Begleitung zentral. [...] Die vorliegende Handreichung will christliche Seelsorgerinnen und Seelsorger bei dieser Aufgabe unterstützen, indem sie:

\section{-}

67 Vgl. Christoph Sigrist, Diakonik im entkonfessionalisierten Kontext, in: Eurich / Schmidt (Hg.), Diakonik (Anm. 7), 366-387.

68 Reformierte Kirche Bern-Jura-Solothurn u.a. (Hg.), Christlich-muslimische Trauerfälle, Bern 2016 
- Wichtige Elemente des muslimischen Verständnisses vom Sterben und von der Zeit danach darstellt;

- Herausforderungen skizziert, mit denen sich Musliminnen und Muslime in der schweizerischen Gesellschaft beim Sterben und im Todesfall auseinandersetzen müssen;

- Aufgaben und Perspektiven für christliche Seelsorgerinnen und Seelsorger bei der Begleitung muslimisch-christlicher Trauerfälle aufzeigt.. ${ }^{69}$

Die im Kanton Bern entwickelte Broschüre - und dies ist auch typisch schweizerisch - wird nun in den übrigen Kantonen auf je unterschiedliche Weisem in ihre kirchliche Kultur aufgenommen und weiterentwickelt.

Mit dem Blick hin zur Arbeit von christlichen Seelsorgenden an der muslimischen Bevölkerung wird noch einmal deutlich, dass im komplexen Zusammenspiel von Organisationen, Kirchen, Religionen und Kulturen die Wirkung helfenden Handelns im Letzten im Verhalten des Einzelnen eingelagert ist. Aus christlicher Sicht bleibt das Erbe der Reformation auch in den aktuellen und zukünftigen Transformationen für einzelne wie auch für kirchliche Gemeinschaften, Institutionen und diakonische Werke handlungsleitend. Dabei ist der Faden Zwinglis wohl nicht nur auf den Stoff einer Zürcher- oder Schweizerfahne immer wieder einzuweben, um je ein eigenes Muster daraus zu entwerfen:

«Ein Christ syn ist nit schwätzen von Christo, sunder wandlen, wie er gewandelt hat. Ein Christ syn ist der schönst und zierlichest Adel, der in dem Himmel und uff erden sin mag.»

69 Reformierte Kirchen Bern-Jura-Solothurn u.a., Trauerfälle (Anm. 68), 5 f.
Autor:

Christoph Sigrist,

PD Dr. theol., Dozent für Diakoniewissenschaft an der Universität Bern und Pfarrer am Grossmünster in Zürich 